\title{
Benign muscular dystrophy: risk calculation in families with consanguinity*
}

\author{
G WOLFF†, C R MÜLLER , ANDT GRIMM \\ From the Department of Human Genetics, University of Freiburg i Br; and $\ddagger$ the Department of Human \\ Genetics, University of Würzburg, Federal Republic of Germany.
}

SUMmARY This report concerns two families in which the index patients are sporadic cases of a benign form of muscular dystrophy. In both families the sisters of the patients have married a close relative. The respective risks for a child of these consanguineous marriages being affected with either X linked Becker muscular dystrophy or autosomal recessive limb girdle muscular dystrophy is calculated using pedigree information, results of serum creatine kinase determinations, and also, in one family, results of DNA typing using RFLPs from the short arm of the $\mathrm{X}$ chromosome.

The Becker type of $\mathrm{X}$ linked muscular dystrophy (BMD) often has a moderate course which makes it clinically indistinguishable from the early manifestation of autosomal recessive limb girdle muscular dystrophy (LGMD). ${ }^{1}$ This similarity leads to problems in genetic counselling when the family history is not typical of $\mathrm{X}$ linkage or when one is dealing with a sporadic male patient. ${ }^{2}$ In both families described here, the counsellees are sisters of a sporadic case and have married a close relative (second cousin), so it became crucial to estimate the recurrence risks both for $\mathrm{X}$ linked and for autosomal recessive inheritance. For the purpose of risk estimation for female relatives of such cases, one has to start from a prior probability that the mother of an affected male is heterozygous for BMD. Alternatively, one has to take into consideration the probability that both parents may be heterozygous for LGMD. These prior probabilities have been derived from the mutation rate of BMD and the relative frequencies of $\mathrm{BMD}$ and LGMD. $^{3}$

Beginning with these prior probabilities and using the Bayesian principle, one can calculate the risk for the counsellee having a child affected with either BMD or LGMD for each family situation including cases of consanguinity.

\section{Case reports}

FAMILY 1 (FIG 1)

In this family, the sister (C) of an affected male

* This paper is dedicated to Professor P E Becker on the occasion of his 80th birthday on 23 November 1988.

Received for publication 15 February 1988.

Revised version accepted for publication 11 October 1988.

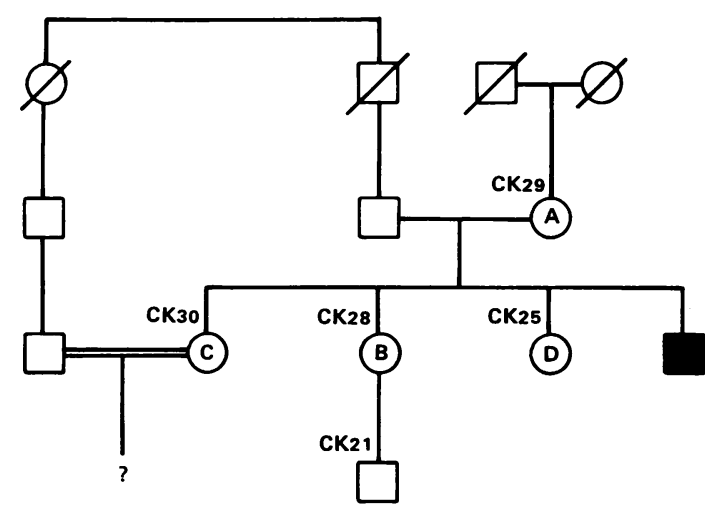

FIG 1 Pedigree of family 1.CK values are the median of at least three estimations (upper limit for normal values $70 \mathrm{IU})$.

presented for genetic counselling. The family history showed no evidence of any further affected male or female relatives. The counsellee and her husband were second cousins. On the basis of the available data and the clinical course in the affected brother, we could not be certain whether we were dealing with BMD or LGMD. Neither the mother (A), the counsellee (C), nor her two sisters (B and D) showed any clinical signs of muscular disorder. The mean values of repeated serum creatine kinase (CK) determinations were within the normal range for all four females.

FAMILY 2 (FIG 2)

In the second family the counsellee (C) had one 


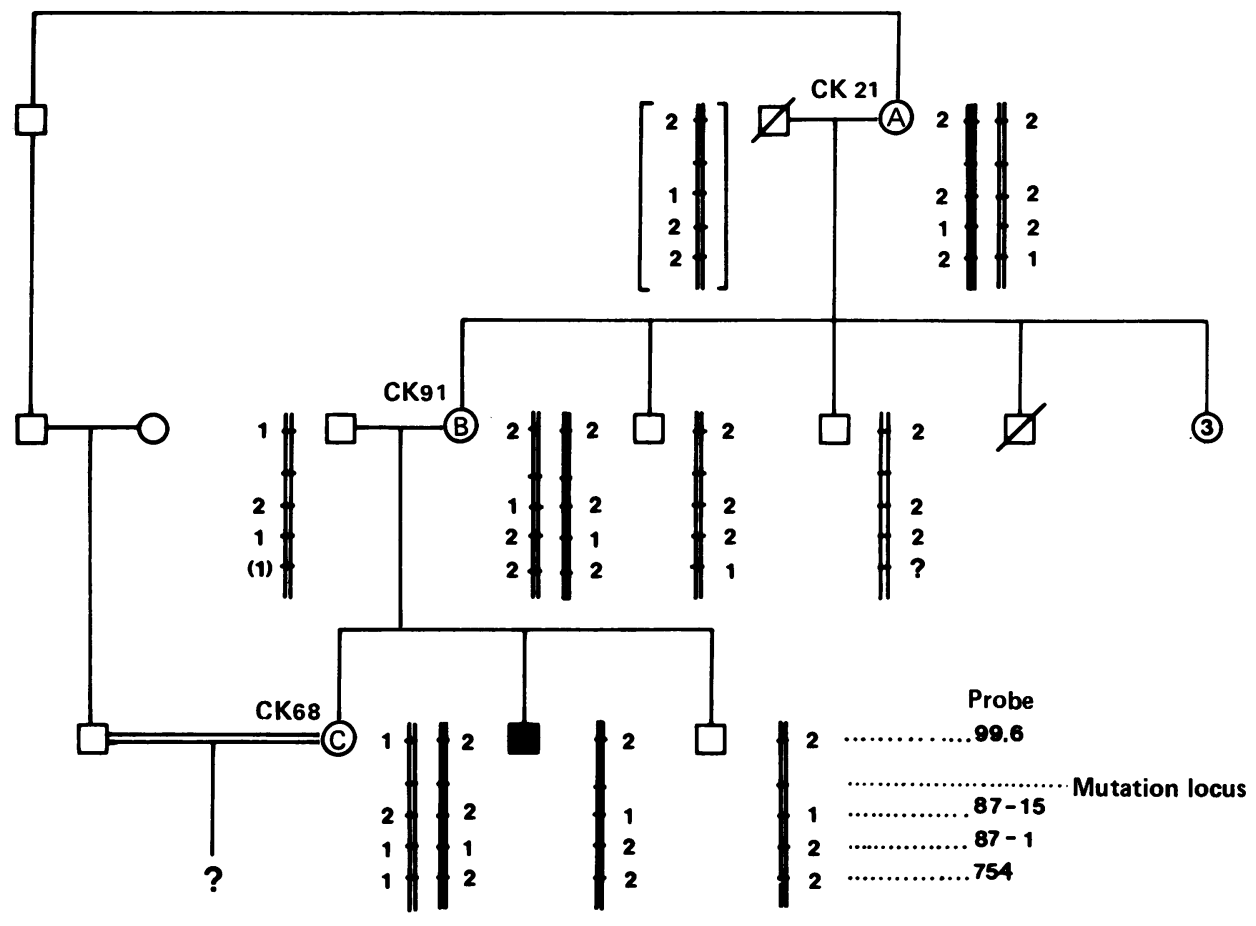

FIG 2 Pedigree of family 2. CK values are the median of multiple estimations (upper limit for normal values 70 IU). Included is the most plausible explanation for the segregation of RFLPs from the short arm of the X chromosome with a crossover between the mutation locus and the pERT87-15 marker in the meiosis that led to the index patient if he has BMD.

affected and one unaffected brother. The affected brother had the clinical manifestations of a benign muscular dystrophy. The pedigree was not helpful in distinguishing between $\mathrm{X}$ chromosomal and autosomal recessive inheritance. In this family, too, the counsellee and her husband were second cousins. Testing for serum CK showed slightly increased values in the counsellee (C) and her mother (B). The grandmother (A) had normal CK values.

\section{Results}

For both counsellees we calculated the respective risks of having a child affected with BMD or LGMD or either of these two disorders.

FA M I L Y 1

Calculations of risk were prepared first under the assumption that the affected brother suffers from BMD. Using information from the pedigree alone, the probability that counsellee $(\mathrm{C})$ is heterozygous for BMD is $43.5 \%$ and her risk of having a boy affected with BMD is, therefore, $21 \cdot 8 \%$. As the CK values in all the female relatives tested were normal, her risk of having an affected son is reduced to $0.3 \%$.

Thus, the probability of the counsellee (C) being heterozygous for BMD is relatively low. This probability would increase to only about 1 to $2 \%$ if DNA haplotyping showed that the counsellee had inherited the same $\mathrm{X}$ chromosome from her mother as her affected brother. As this result would have little impact on the counsellee's family planning, she decided against DNA analysis.

Alternatively, calculations of risk were prepared under the assumption that the affected brother suffers from LGMD. In this case the probabilities of the counsellee $(\mathrm{C})$ and her husband being heterozygous for LGMD are $2 / 3$ and $1 / 16$, respectively. Their risk of having a child with LGMD is, therefore, about $1 \%$.

We next prepared calculations of risk for a child of the counsellee having either BMD or LGMD, under the assumption that the index patient has one or the other disease (appendix). This calculation takes into account the relative frequencies of the 
two diseases. However, one problem in this calculation arises from interpretation of $\mathrm{CK}$ values in heterozygotes for LGMD. We could not find reliable published data regarding the distribution of CK values in LGMD heterozygotes and we can only speculate on a possible effect of the heterozygous state in LGMD on the serum CK level. Therefore, we considered two models in the calculations of risk based on different assumptions of the effect of heterozygosity on serum CK values. (1) The first model assumes that the CK values of heterozygotes for LGMD are in the normal range. ${ }^{45}$ (2) The second model assumes that $50 \%$ of LGMD heterozygotes have raised $\mathrm{CK}$ values.

The application of the first model results in an approximately three times higher probability for LGMD than BMD in the index patient. The risk for the counsellee having a child affected with either BMD or LGMD is $0.8 \%$.

Using the second model, the presence of normal CK values for all females tested lowers the probability of their being heterozygous for LGMD. Therefore, the relative probability of the brother being affected with BMD is correspondingly increased two-fold. However, the normal $C K$ values in all females also argue in favour of a new mutation for BMD in the affected brother. Therefore, the risk for the counsellee having a child affected with either BMD or LGMD is only $0.3 \%$. The results of these calculations are shown in the table.

FAMILY 2

If the index patient had BMD, the probability of the counsellee (C) being heterozygous for BMD would be $21.9 \%$ and the corresponding risk of having an affected male child $11.0 \%$, when based on the pedigree information alone. When serum CK values are also considered (the normal CK of the grandmother (A), the increased CK values of the mother (B), and the slightly raised $\mathrm{CK}$ values of the counsellee (C)), the risk of the counsellee having an affected son is increased to $29.8 \%$.

Because of this high risk, we performed DNA analysis in this family using the DMD-cDNA for deletion screening ${ }^{6}$ and $X$ specific DNA markers that have been shown to be linked to the BMD locus. ${ }^{7}$ A molecular deletion within the Duchenne/ Becker gene was not detectable (data not shown). The results of the DNA haplotyping are shown in fig 2 . The mother (B) is heterozygous for intragenic RFLPs as indicated by the DNA probes pERT87-15 and pERT $87-1 .{ }^{8}$ Both the healthy and the affected brother have inherited the same $\mathrm{X}$ chromosomal haplotype from their mother and the counsellee the other one. However, the probability of the counsellee being heterozygous for BMD remains nearly unchanged when the information from the DNA analysis is added to the data provided by the pedigree and CK determinations (59.6\% v 53.5\%).

The DNA data can be explained either by a new mutation in the index patient or by a crossover between the pERT87 markers and the mutation locus. In the latter case, it is not possible to pinpoint this crossover to any one of the $\mathrm{X}$ chromosomes. The raised CK values of females (B) and (C) are in favour of a crossover event in the maternal meiosis that led to the index patient.

If we start from the diagnosis of LGMD the

TABLE Posterior probabilities for BMD and LGMD, respectively, calculated using different levels of conditional information. Pedigree: only pedigree information is used; CK: odds for being carrier based on serum CK levels ${ }^{4}$ DNA: information from DNA haplotypes is included. The two models for the distribution of CK levels in heterozygotes for LGMD are explained in the text.

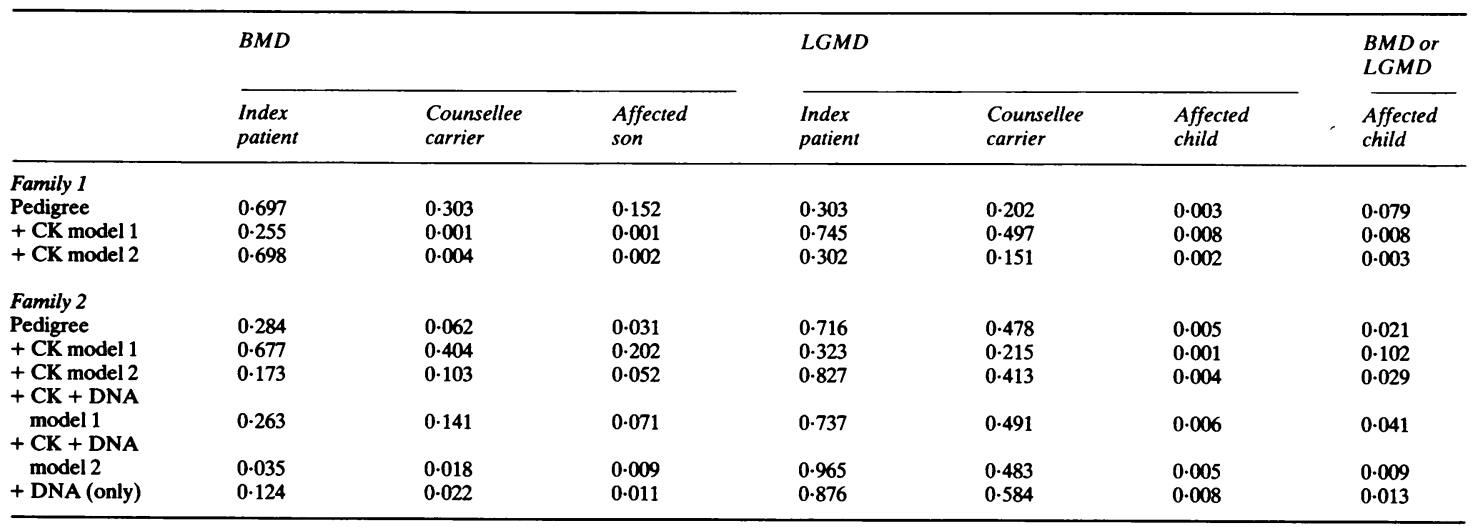


probability of the counsellee $(\mathrm{C})$ and her husband being heterozygous for LGMD is $2 / 3$ and $1 / 16$, respectively; therefore, the risk of having a child with LGMD is about $1 \%$.

Next we calculated the risk of a child of the counsellee being affected with either BMD or LGMD under the assumption that the affected brother must have either one or the other disease.

As in family 1 , this calculation takes into account the relative frequencies of the two diseases as well as the two models of distribution of $\mathrm{CK}$ values in heterozygotes of LGMD (appendix).

Application of the first model results in a probability about three times higher for LGMD than BMD in the index patient. The risk for the counsellee having a child affected with either BMD or LGMD is $4 \cdot 1 \%$. In the second model, the normal and the increased CK values of the females lower the probability of the affected brother having BMD $(3.5 \%)$. The combined risk for the counsellee having a child affected with either BMD or LGMD is only $0.9 \%$. The results of all calculations are shown in the table.

\section{Discussion}

In families affected with a benign muscular dystrophy, requests for genetic counselling usually come from the sisters of the index patients rather than from their mothers. In this situation, besides the risk for $\mathrm{X}$ linked $\mathrm{BMD}$, one also has to consider the risk for autosomal recessive LGMD, particularly if the counsellee is related to her partner. In such families, risk calculation must take into account not only the risk for BMD but for LGMD as well. This calculation can be done using the Bayesian principle. The prior probabilities are the heterozygote frequencies of BMD and LGMD. ${ }^{2}$

As conditional probabilities, pedigree information, CK values of females, or results from DNA analyses can be used (appendix). At present, detailed molecular analysis using either the $\mathrm{DMD} /$ BMD-cDNA or linked DNA markers is available only for the $\mathrm{X}$ linked form. The observation of a deletion within the DMD/BMD gene can be regarded as decisive for the differential diagnosis in question. Therefore, deletion screening in sporadic patients with benign muscular dystrophy represents the most powerful diagnostic tool at present. If no deletion is observed, the decision as to when to do DNA haplotyping for linked markers in a given family depends on the posterior probability of the counsellee being heterozygous for $\mathrm{BMD}$, taking into account that the affected brother has either BMD or LGMD.
In the absence of an $\mathrm{X}$ chromosomal deletion, in families with a sporadic case of benign muscular dystrophy, CK values of female relatives are critical for the risk calculation, but nothing is known about the distribution of CK values in LGMD heterozygotes. Therefore, we made two assumptions about a possible effect of heterozygosity for LGMD on the serum CK levels. The results in our two families show that if some of the heterozygotes for LGMD have increased CK values, any distribution of these CK values would have a considerable influence on the likelihood of the index patient having BMD or LGMD. However, with respect to the risk of the counsellee having an affected child, there is only a slight effect which may be negligible in practice.

In families in which the female relatives have normal CK values, the combined risk is determined largely by the risk for LGMD, which is low.

In families with increased CK levels, in some females the combined risk is predominated by the risk for BMD and independent of the distribution of CK values in heterozygotes for LGMD. In our family 2, no deletion within the DMD/BMD gene has been found. Moreover, if the index patient indeed had BMD, a new mutation or a recombination of the maternal $\mathrm{X}$ chromosomes must be postulated (fig 2). These three factors lower the relative probability for $\mathrm{BMD}$.

\section{References}

${ }^{1}$ Emery AEH. Muscular dystrophy, benign X-linked (Becker). In: Vinken PJ, Bruyn GW, eds. Handbook of clinical neurology. Vol 43. Amsterdam: North-Holland, 1982:86-8.

2 Young ID, Nugent Z, Grimm T. Autosomal or sex linked recessive: a counselling dilemma. J Med Genet 1986;23:32-4.

${ }^{3}$ Grimm T. Genetic counseling in Becker type X-linked muscular dystrophy. I. Theoretical considerations. Am J Med Genet 1984;18:713-8

${ }^{4}$ Grimm T. Genetic counseling in Becker type X-linked muscular dystrophy. II. Practical considerations. Am J Med Genet 1984;18:719-23.

5 Skinner R, Emery AEH, Anderson AJB, Foxall C. The detection of carriers of benign (Becker type) $\mathrm{X}$-linked muscular dystrophy. J Med Genet 1975;12:131-4.

${ }^{6}$ Koenig M, Hoffman EP, Bertelson CJ, Monaco AP, Feener C, Kunkel LM. Complete cloning of the Duchenne muscular dystrophy (DMD) cDNA and preliminary genomic organization of the DMD gene in normal and affected individuals. Cell 1987;50:509-17.

7 Bakker E, Goor N, Wrogemann K, et al. Prenatal diagnosis and carrier detection of Duchenne muscular dystrophy with closely linked RFLPs. Lancet 1985; i:655-8.

${ }^{8}$ Monaco AP, Bertelson CJ, Middlesworth W, et al. Detection of deletions spanning the Duchenne muscular dystrophy locus using a tightly linked DNA segment. Nature 1985;316:842-5.

Correspondence to $\mathrm{Dr} \mathrm{G}$ Wolff, Institut für Humangenetik, Albertstrasse 11, D-7800 Freiburg i $\mathrm{Br}$, Federal Republic of Germany. 
APPENDIX Complete sequence of risk calculations in both families

The Bayesian principle is used for the calculation of the different posterior probabilities. The prior probabilities are the heterozygote frequencies for BMD and LGMD. Both can be expressed in terms of the mutation rate in BMD, $18 \mu$ and $32 \mu$, respectively. ${ }^{4}$ As conditional probabilities, pedigree information, the odds of $\mathrm{CK}$ values of females, or the DNA haplotypes can be used.

\section{Risk calculation in family 1}

Pedigree information (fig 1): the counsellee (female C) has one affected brother (BMD or LGMD); there is no other family history of muscular dystrophy. The CK levels in the females of this family are (median of multiple estimations):

\begin{tabular}{|c|c|c|c|c|}
\hline \multirow{2}{*}{$\begin{array}{l}\text { Female } \\
\text { (age) }\end{array}$} & \multirow{2}{*}{$\begin{array}{l}C K \\
(I U / l)\end{array}$} & \multirow[t]{2}{*}{ Odds (BMD) } & \multicolumn{2}{|c|}{ Odds $(L G M D)^{*}$} \\
\hline & & & Model 1 & Model 2 \\
\hline A $(52 y)$ & 29 & $\mathrm{ha}=1 / 7.9$ & $\mathbf{k a}=1$ & $1 / 2$ \\
\hline B (27 y) & 28 & $h b=1 / 26 \cdot 7$ & $k b=1$ & $1 / 2$ \\
\hline C (24 y) & 30 & $\mathrm{hc}=1 / 25 \cdot 3$ & $\mathrm{kc}=1$ & $1 / 2$ \\
\hline D $(21$ y) & 25 & $\mathrm{hd}=1 / 44 \cdot 0$ & $\mathrm{kd}=1$ & $1 / 2$ \\
\hline
\end{tabular}

*Model 1: normal CK values in heterozygotes; model 2: increased CK values in $50 \%$ of heterozygotes.
We wanted to know the risk of the woman (C) being a carrier for $\mathrm{BMD}$ ( $\left.\mathrm{P}_{\mathrm{C}-\mathrm{BMD}}\right)$ or heterozygous for LGMD $\left(\mathrm{P}_{\mathrm{C}-\mathrm{LGMD}}\right)$, where $\mathrm{C}=$ carrier of $\mathrm{BMD}$; $\mathrm{NC}=$ not carrier of $\mathrm{BMD} ; \mathrm{Het}=$ heterozygote for LGMD; Het-Het=the female and her husband are heterozygotes for LGMD; $\mu=$ mutation rate in BMD; as=affected son; hs=healthy son.

\section{Risk calculation in family 2}

Pedigree information (fig 2): the counsellee (C) has one affected brother (BMD or LGMD); there is no other family history of muscular dystrophy. The CK levels in the females of this family are:

\begin{tabular}{lllll}
\hline $\begin{array}{l}\text { Female } \\
\text { (age) }\end{array}$ & $\begin{array}{l}\text { CK } \\
\text { (IU/l) }\end{array}$ & Odds $(B M D)$ & \multicolumn{2}{l}{ Odds $($ LGMD)* } \\
\cline { 4 - 5 } & & & Model 1 & Model 2 \\
\hline A (79 y) & 21 & ha $=1 / 4 \cdot 5$ & ka=1 & $1 / 2$ \\
B (46 y) & 91 & hb $=1 / 0 \cdot 1$ & $k b=1$ & $1 / 0 \cdot 05$ \\
C (20 y) & 68 & hc=1/0.5 & kc=1 & $1 / 2$ \\
\hline
\end{tabular}

${ }^{*}$ Model 1: normal CK values in heterozygotes; model 2: increased CK values in $50 \%$ of the heterozygotes.

The DNA haplotypes are shown in fig 2.

We wanted to know the risk of the woman (C) being a carrier for $\mathrm{BMD}\left(\mathrm{P}_{\mathrm{C}-\mathrm{BMD}}\right)$ or heterozygous

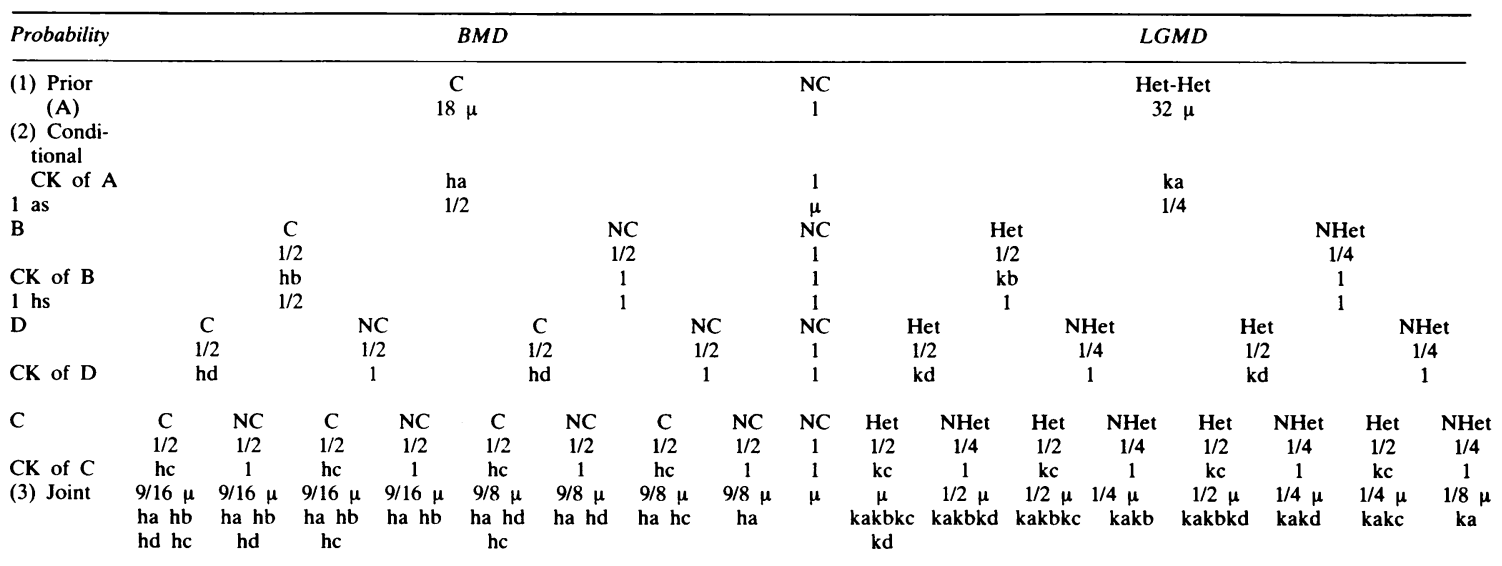

(4) Posterior probability (P):

$$
\begin{aligned}
& \text { P }- \text { BMD }=\frac{9 / 16 \mu \text { ha hc (hb hd }+ \text { hb }+2 \text { hd }+2)}{9 / 16 \mu \text { ha (hc hb hd }+ \text { hb hd }+ \text { hb hc }+ \text { hb }+2 \text { hd hc }+2 \text { hd }+2 \text { hc }+2)+\mu+} \\
& +k a \mu(k b k c k d+1 / 2 k b k d+1 / 2 k b k c+1 / 4 k b+1 / 2 k c k d+1 / 4 k d+1 / 4 k c+1 / 8)
\end{aligned}
$$

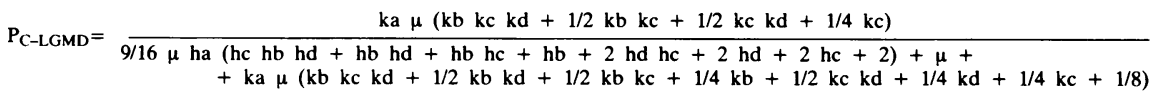


for $\mathrm{LGMD}\left(\mathrm{P}_{\mathrm{C}-\mathrm{LGMD}}\right)$, where $\mathrm{C}=$ carrier of $\mathrm{BMD}$; $\mathrm{NC}=$ not carrier of $\mathrm{BMD} ; \mathrm{Het}=$ heterozygote for LGMD; Het-Het=the female and her husband are heterozygotes for LGMD; NHet=not heterozygous for LGMD; $\mu=$ mutation rate in $\mathrm{BMD}$; as=affected son; hs $=$ healthy son; $\theta=$ recombination fraction between BMD locus and DNA marker (pERT87-1) $(=0.05) ; \mathrm{N}=$ normal allele; $\mathrm{D}=$ defective allele of BMD; 1 and 2 are alleles of the DNA marker (pERT87); $\delta \approx 1 / 2$ if deletion screening (DMD/ BMD gene) is negative and $\delta=1$ if deletion screening is not done.

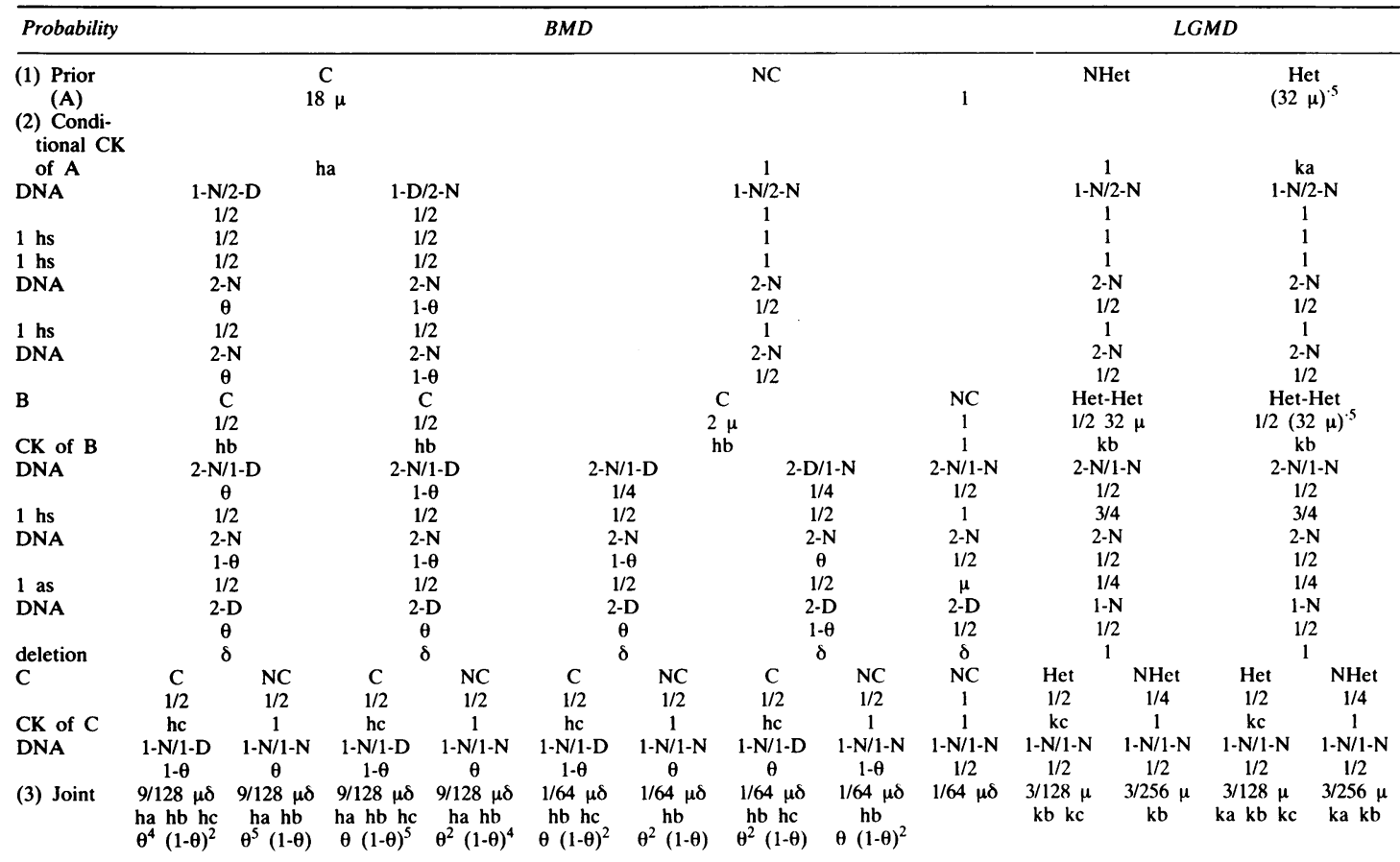

(4) Posterior probability (P):

$9 / 128 \mu \delta$ ha hb he $\theta(1-\theta)^{2}\left(\theta^{3}+(1-\theta)^{3}\right)+1 / 64 \mu \delta$ hb he $\theta(1-\theta)$

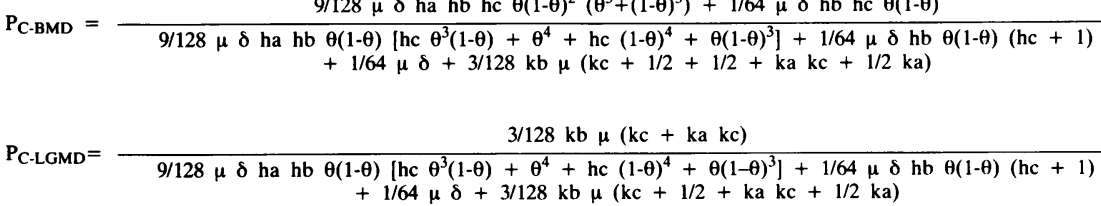

